Rev. Elev. Méd. Vét. Pays trop., 1976, 29 (1) $\cdot 43-58$

\title{
L'élevage de Glossina palpalis gambiensis Vanderplank, 1949 (Diptera-Muscidae) à Maisons-Alfort
}

\author{
par J. ITARD *
}

\begin{abstract}
RÉSUMÉ
Un élevage de Glossina palpalis gambiensis a débuté, en juillet 1972, au laboratoire d'entomologie de l'l. E. M. V. T., à Maisons-Alfort (France) à partir de 126 femelles parentales issues de pupes et femelles sauvages récoltées en Haute-Volta.

Cet élevage a été créé dans le but d'étudier, en laboratoire, la biologie de cette espèce et les conditions de production en élevage de masse, en vue d'une application, en Haute-Volta, de la technique de lutte par lâchers de mâles stériles.

L'auteur fait le point des résultats obtenus depuis l'origine de l'élevage jusqu'en septembre 1975. La souche a été en "phase d'expansion " pendant 2 ans (août 1972-août 1974), avec un coefficient d'accroissement moyen de 0,00672 . Depuis août 1974 , les effectıfs sont maintenus à un niveau moyen constant de 4800 femelles environ, par retrait des pupes ou adultes en excédent (3 000 pupes et 180 femelles environ par mois).

Ces résultats permettent de faire des prévisions pour l'établissement d'un élevage de masse de cette espèce en Afrique.
\end{abstract}

\section{INTRODUCTION}

Des élevages de glossines sont entrepris, depuis août 1964, au laboratoire d'Entomologie de l'Institut d'Elevage et de Médecine Vétérinaire des Pays tropicaux, à Maisons-Alfort (France). Ces élevages permettent de disposer d'un important surplus d'insectes à l'état pupal ou à l'étatadulte, utilisables pour des études diverses et, en particulier, pour la mise au point de techniques de lutte génétique (7).

Les méthodes utilisées dans ce laboratoire sont maintenant entrées dans une phase de routine et permettent, avec un personnel réduit, mais bien entraîné et consciencieux, de manipuler chaque jour près de 30000 insectes.

(*) 1. E. M. V. T., Service Entomologie, 10, rue Pierre-Curie, 94700 Maisons-Alfort, France.
5 espèces différentes sont actuellement élevées :

G. m. morsitans (début de l'élevage : mars 1965),

G. tachinoides (début de l'élevage : avril 1965),

G. austeni (début de l'élevage : novembre 1966),

G. f. fuscipes (début de l'élevage: juin 1968),

G. p. gambiensis.

L'élevage de la dernière espèce, Glossina palpalis gambiensis, a débuté en juillet 1972, à partir de 2 lots de femelles sauvages et de pupes récoltées dans la région de Bobo-Dioulasso (Haute-Volta), où l'I. E. M. V. T. vient d'achever la construction d'un centre de recherche et d'application de la technique du lâcher de mâles stériles. Ce centre a pour premier objectif d'étudier sur le terrain les modalités pratiques d'utilisation de cette technique de lutte et son efficacité contre Glossina palpalis gambiensis, espèce dominante dans cette région (3). 
Un élevage de G.p.gambiensis a, en conséquence, été créé à Maisons-Alfort, dans le but d'étudier, en laboratoire, d'une part la biologie de cette espèce et les conditions de production en élevage de masse, d'autre part de disposer d'un surplus d'insectes permettant, éventuellement, d'alimenter l'insectarium de Bobo-Dioulasso, et enfin de définir les doses optimales d'irradiation gamma applicables aux mâles de cette espèce.

Dans cet article, nous ferons le point des résultats obtenus avec l'élevage en laboratoire de cette souche, qui a été en "phase d'expansion» d'août 1972 à août 1974, et qui est maintenue, depuis cette époque, à un niveau moyen constant par retrait des pupes ou adultes en excédent.

\section{TECHNIQUES D'ÉLEVAGE}

Les conditions générales d'élevage utilisées à Maisons-Alfort et appliquées aux 5 espèces maintenues dans ce laboratoire ont été décrites, pour l'essentiel, dans différents articles parus précédemment $(8,5,6)$.

Rappelons simplement que les adultes sont exclusivement nourris sur lapins, 6 jours par semaine. Des lots de 9 lapins sont utılisés chaque jour, sauf le dimanche, et restent au repos les 6 jours suivants. La nourriture des glossines nécessite $4 \mathrm{~h} 30$ de travail par jour (de 8 h à $12 \mathrm{~h} \mathrm{30}$ ) bien que chaque cage ne soit maintenue sur l'oreille du lapin que pendant 4 à $5 \mathrm{mn}$.

Les femelles sont groupées, par lots de 10 cages de type Roubaud $(14 \times 8,5 \times 5 \mathrm{~cm})$ contenant chacune de 30 à 35 individus, dans des appareils métalliques sous lesquels repose un tiroir garni d'une feuille de tissu de papier. Les larves pondues tombent dans le tiroir où elles se transforment en pupes.

Les femelles reproductrices de G.p. gambiensis sont maintenues, sur des étagères garnissant une extrémité de la salle de stockage, à une température de $25^{\circ} \mathrm{C} \pm 1{ }^{\circ} \mathrm{C}$ et une humidité relative d'environ 80 p. 100 ( \pm 5 p. 100).

Les jeunes femelles sont accouplées à l'âge de 3 à 4 jours avec des mâles âgés de 7 à 10 jours, à raison de 20 femelles pour 15 mâles. Les sexes sont séparés au bout de $48 \mathrm{~h}$.

Les pupes, récoltées chaque matin, sont aussitôt placées dans des tubes de verre stériles de $8 \mathrm{~cm}$ de hauteur sur $3,6 \mathrm{~cm}$ de diamètre, sans sable, à raison de 30 à 35 pupes par tube. Ces tubes, recouverts d'un carré de tulle de tergal maintenu par un bracelet de caoutchouc, sont stockés jusqu'à éclosion dans une petite pièce à $24^{\circ} \pm 1^{\circ} \mathrm{C}$ et à $80-85$ p. 100 d'H. R.

\section{L'ELEVAGE EN PHASE D'EXPANSION}

\section{GENÉRATION PARENTALE}

Les deux lots de pupes et de femelles sauvages de G.p.gambiensis récoltés en Haute-Volta par notre confrère, le Dr Vétérinaire CLAIR, ont été reçus à Maisons-Alfort le 30 juin et le 17 juillet 1972. Le premier lot comprenait à l'arrivée 37 femelles vivantes et 20 pupes; le second lot, 58 femelles vivantes et 76 pupes.

Les femelles sauvages ont eu une longévité moyenne, au laboratoire, de 35,2 jours et ont pondu 201 pupes, ayant donné naissance à 95 mâles et 92 femelles (pourcentage d'éclosion = 93,03 p. 100 ).

Les pupes pondues au laboratoire par les femelles sauvages du premier lot pesaient en moyenne $22,45 \mathrm{mg} \pm 0,73 \mathrm{mg}$. Le poids des pupes pondues, au laboratoire, par les femelles sauvages du second lot a été de $21,01 \mathrm{mg} \pm$ $0,78 \mathrm{mg}$ (différence significative à 1 p. 100 : $\varepsilon=2,71$ ).

Les durées de nymphose des individus issus de ces pupes ont été de $38,2 \pm 0,6$ jours pour les femelles et de $40,7 \pm 0,5$ jours pour les mâles.

Les pupes sauvages ont donné naissance, au laboratoire, entre le 24 juillet et le 28 août, à 25 mâles et 34 femelles viables (pourcentage d'éclosion $=61,45$ p. 100).

L'ensemble de ces individus, éclos au laboratoire, a constitué la génération parentale.

La mortalité des femelles non reproductrices (ensemble des femelles mortes entre l'éclosion et la séparation des sexes après l'accouplement), a été de 22,07 p. 100.

La longévité maximale des femelles reproductrices a atteint 160 jours. Plus de la moitié d'entre elles ont vécu au-delà de 40 jours. La production moyenne a été de 3 pupes par femelle reproductrice.

Les pupes pesaient en moyenne $22,16 \pm 0,39 \mathrm{mg}$. Il n'y a pas de différence significative $(\varepsilon=1,071)$ entre le poids des pupes 
mâles $(m=21,94 \pm 0,56 ; n=142)$ et celui des pupes femelles $(m=22,36 \pm 0,54 ; n=159)$.

Les durées de nymphose des individus de première génération issus de ces pupes ont été de $34,6 \pm 0,5$ jours pour les femelles et de $36,7 \pm 0,6$ jours pour les mâles, soit en moyenne 4 jours de moins que pour la génération parentale (différences très hautement significatives: $\varepsilon=8,84$ pour les femelles et 10,00 pour les mâles). Les conditions d'élevage en laboratorre (température et humidité constantes) expliquent ce raccourcissement de la durée de nymphose.

\section{COMPORTEMENT GÉNERAL DE L'ÉLEVAGE}

Une population animale soumise à des conditions constantes, sans qu'aucune limitation ne soit imposée à sa croissance, avec une longévité et une fécondité moyenne non affectées par les modifications de la densité, approchera d'une forme fixe de répartition des âges. Lorsque la proportion des différents âges est constante, la population aura un taux de croissance exponentiel mesuré par la fonction :

$$
N_{t}=N_{0} e^{r_{m} t}
$$

où : $\mathrm{N}_{0}$ est l'effectif au temps o,

$N_{t}$, l'effectif au temps $t$,

$r_{m}$, le coefficient naturel d'accroissement dans les conditions d'environnement auxquelles est soumise la population.

La souche de G. p. gambiensis de MaisonsAlfort semble s'être adaptée d'emblée aux conditions du laboratoire. La progression des effectifs des femelles, bien que relativement lente, a été régulière. Les productions de pupes ont également été en progression constante (graphique 1).

L'effectif moyen, qui était, en août 1972, de 54 femelles, a atteint son minimum ( 47 femelles) en octobre 1972 . Il a ensuite progressé régulièrement, atteignant une moyenne mensuelle de 94 femelles en janvier 1973, 1300 femelles en janvier 1974 et 4700 femelles en août 1974 (tabl. I et graphique 1).

Les productions mensuelles de pupes sont passées de 70 pupes en octobre 1972 à 185 pupes en janvier 1973, 1850 pupes en janvier 1974 et 7300 pupes en août 1974 .

A partir du 10 janvier 1973 (effectif des femelles $\mathrm{N}_{0}=100$ ) jusqu'au 14 août 1974 (effectif des femelles $\mathrm{N}_{\mathrm{t}}=4997$ ), soit pendant le temps $t=582$ jours, la population en élevage a été dans une phase de croissance approchant une forme exponentielle.

Le coefficient naturel d'accroissement $r_{m}$, pendant toute cette période, est égal à 0,00672 .

En fait, l'accroissement des effectifs n'a pas suivi rigoureusement une courbe exponentielle. Elle a subi des fluctuations dues à des causes diverses ayant momentanément provoqué soit un accroissement des mortalités, soit une diminution des taux d'éclosion ou de la fécondité des femelles.

Pour citer un exemple précis, une forte mortalité des femelles reproductrices, atteignant 50 p. 100 de l'effectif, s'est produite pendant la période comprise entre le 20 août et le 18 septembre 1973. Cette mortalité a été provoquée par l'utilisation, du 17 au 22 août 1973, pour le lavage du sol de l'insectarium, d'un savon noir parfumé dégageant des vapeurs irritantes. Cette période de mortalité a été suivie d'une diminution du nombre de pupes par femelle, puis d'une baisse du pourcentage d'éclosion. Les répercussions de cette intoxication accidentelle ont été ressenties jusqu'en décembre 1973.

L'analyse de la courbe d'accroissement des effectifs permet ainsi de distinguer cinq périodes pendant lesquelles les taux de croissance ont subi des variations importantes (graphique 2).

La première période, comprise entre août 1972 et janvier 1973, correspond à la phase initiale pendant laquelle le nombre moyen de femelles étant inférieur à 100 , la distribution d'âge de la population ne peut être considérée comme stable.

La seconde période s'étend de fin janvier à fin juillet 1973. Le coefficient d'accroissement atteint une valeur $r_{m}$ égale à 0,0087 .

Pendant la troisième période (fin juillet à midécembre 1973), $r_{m}$ est égal à 0,0053 . C'est pendant cette période que s'est produite l'intoxication signalée précédemment.

Au cours de la $4^{\mathrm{e}}$ période (fin décembre 1973 à mi-mai 1974) $r_{m}$ augmente à nouveau pour atteindre une valeur légèrement supérieure à 0.0067 .

C'est pendant la $5^{e}$ période (mi-mai à mi-août 1974) que $r_{m}$ atteint sa valeur la plus faible $\left(r_{m}=0,0047\right)$. Dès cette époque, des prélèvements de pupes destinées à des expérimentations diverses ont en effet été effectués, à une moyenne d'environ 300 pupes par mois, pendant 4 mois. 
TABLEAU $\mathrm{N}^{\circ} \mathrm{I}-G \cdot p \cdot$ gambiensis - Elevage en phase d'expangion.

\begin{tabular}{|c|c|c|c|c|c|c|}
\hline $\begin{array}{l}\text { Përrodes de } \\
\text { 30 jours } \\
\text { Dates }\end{array}$ & $\begin{array}{l}\text { Nombre } \\
\text { moyen de } \\
\text { femel les }\end{array}$ & $\begin{array}{l}\text { Production } \\
\text { de pupes }\end{array}$ & $\begin{array}{l}\text { Pourcentage } \\
\text { d'éclosion }\end{array}$ & $\begin{array}{l}\text { Pupes par } \\
\text { femelle en } \\
30 \text { jours }\end{array}$ & $\begin{array}{c}\text { Mortalité } \\
\text { journalière-p. } 100 \\
\text { du nombre moyen } \\
\text { de femelles }\end{array}$ & $\begin{array}{l}\text { Pupes ou } \\
\text { adultes } \\
\text { prélevés }\end{array}$ \\
\hline $\begin{array}{l}26.7 .72 \\
24 . \quad 8.72 \\
\end{array}$ & 54 & 67 & 92,53 & 1,24 & 2,77 & ' \\
\hline $\begin{array}{l}25.8 .72 \\
23.9 .72 \\
\end{array}$ & 59 & 93 & 94,93 & 1,58 & 2,43 & 14 pupes \\
\hline $\begin{array}{r}24.9 .72 \\
23.10 .72 \\
\end{array}$ & 47 & 70 & 94,20 & 1,49 & 3,04 & \\
\hline $\begin{array}{r}24.10 .72 \\
22.11 .72 \\
\end{array}$ & 53 & 75 & 95,94 & 1,41 & 2,18 & \\
\hline $\begin{array}{l}23.11 .72 \\
22.12 .72 \\
\end{array}$ & 65 & 132 & 95,45 & 2,01 & 1,62 & \\
\hline $\begin{array}{r}23.12 .72 \\
21.11 .73 \\
\end{array}$ & 94 & 185 & 91,35 & 1,97 & 0,77 & \\
\hline $\begin{array}{l}22.1 .73 \\
20,2.73 \\
\end{array}$ & 139 & 261 & 93,87 & 1,87 & 1,31 & \\
\hline $\begin{array}{r}21.2 .73 \\
22.3 .73 \\
\end{array}$ & 177 & 306 & 96,73 & 1,73 & 1,33 & \\
\hline $\begin{array}{l}23 . \quad 3.73 \\
21.4 .73 \\
\end{array}$ & 235 & 412 & 94,41 & 1,75 & 1,23 & \\
\hline $\begin{array}{l}22.4 .73 \\
21.5 .73 \\
\end{array}$ & 303 & 475 & 90,94 & 1,56 & 1,09 & \\
\hline $\begin{array}{l}22.5 .73 \\
20.6 .73 \\
\end{array}$ & 381 & 592 & 95,03 & 1,55 & 1,50 & 8 pupes \\
\hline $\begin{array}{l}21.6 .73 \\
20.7 .73 \\
\end{array}$ & 481 & 646 & 95,63 & 1,34 & 1,29 & 5 pupes \\
\hline $\begin{array}{l}21.7 .73 \\
19.8 .73 \\
\end{array}$ & 542 & 830 & 91,19 & 1,53 & 1,33 & 12 pupes \\
\hline $\begin{array}{l}20.8 .73 \\
18.9 .73 \\
\end{array}$ & 623 & 935 & 91,29 & 1,50 & 2,01 & 4 pupes \\
\hline $\begin{array}{r}19,9.73 \\
18.10 .73 \\
\end{array}$ & 744 & 990 & 93,79 & 1,33 & 0,68 & 7 pupes \\
\hline $\begin{array}{l}19.10 .73 \\
17.11 .73 \\
\end{array}$ & 929 & 1362 & 90,69 & 1,46 & 1,42 & 19 pupes \\
\hline $\begin{array}{l}18.11 .73 \\
17.12 .73 \\
\end{array}$ & 1037 & 1588 & 92,34 & 1,53 & 0,96 & 21 pupes \\
\hline $\begin{array}{l}18.12 .73 \\
16.1 .74 \\
\end{array}$ & 1300 & 1849 & 93,80 & 1,42 & 0,98 & 26 pupes \\
\hline $\begin{array}{l}17.1 .74 \\
15.2 .74 \\
\end{array}$ & 1613 & 2370 & 94,00 & 1,46 & 0,88 & \\
\hline $\begin{array}{ll}16 . & 2.74 \\
17 . & 3.74 \\
\end{array}$ & 1969 & 2919 & 92,27 & 1,48 & 0,88 & 33 q et $60 \mathrm{p}$. \\
\hline $\begin{array}{l}18.3 .74 \\
16.4 .74 \\
\end{array}$ & 2448 & 4062 & 93,12 & 1.66 & 1,29 & 309 pupes \\
\hline $\begin{array}{l}17.4 .74 \\
16.5 .74 \\
\end{array}$ & 3030 & 4777 & 95,89 & 1,57 & 1,20 & 120 pupes \\
\hline $\begin{array}{l}17.5 .74 \\
15.6 .74 \\
\end{array}$ & 3450 & 5516 & 95,46 & 1,59 & 1,22 & 309 pupes \\
\hline $\begin{array}{l}16.6 .74 \\
15.7 .74 \\
\end{array}$ & 4030 & 6402 & 95,80 & 1,58 & 1,33 & 369 pupes \\
\hline $\begin{array}{l}16.7 .74 \\
14.8 .74 \\
\end{array}$ & 4699 & 7285 & 94,63 & 1,55 & 1,63 & 2272 pupes \\
\hline
\end{tabular}




\section{ELEVAGE DE G. P. GAMBIENSIS}

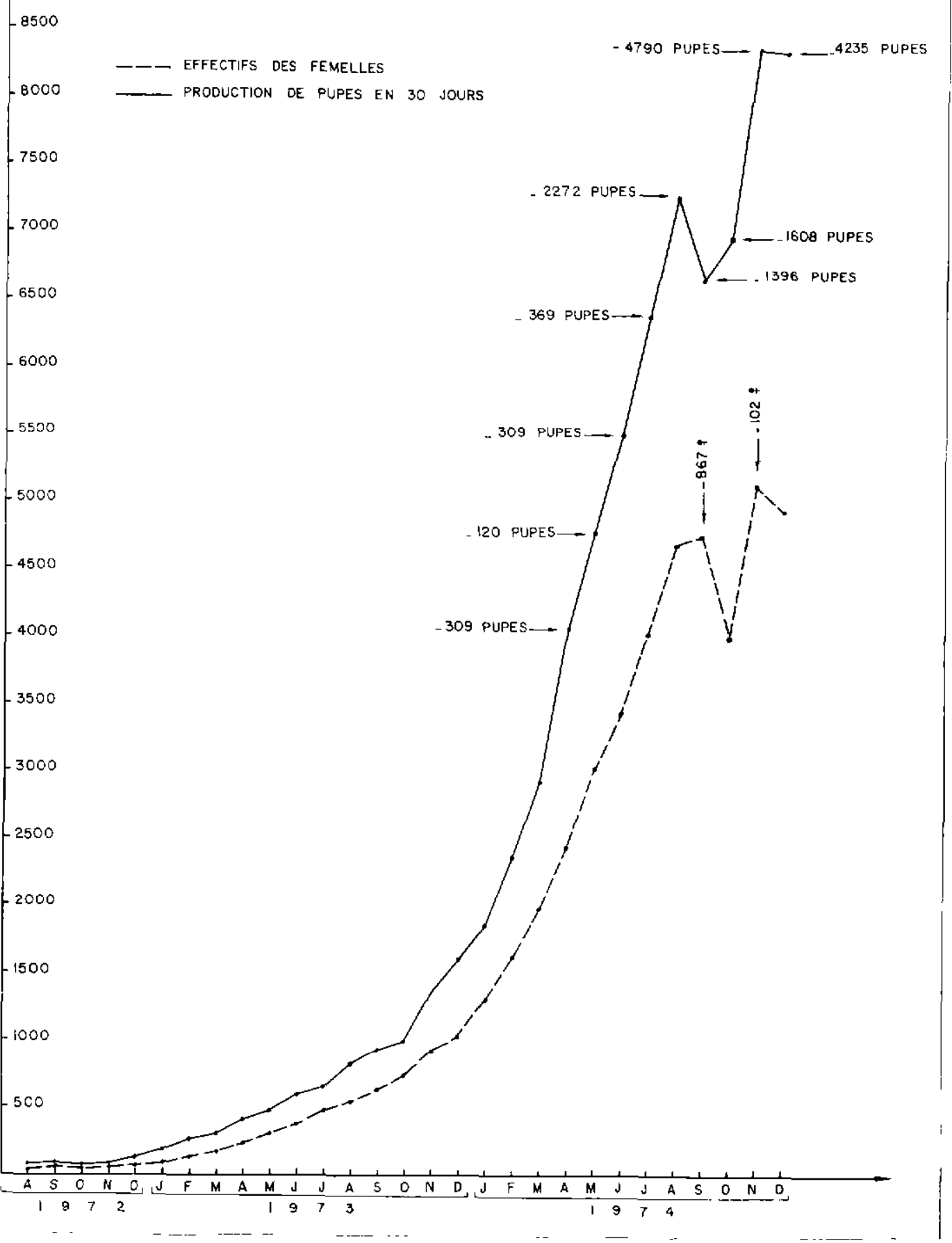




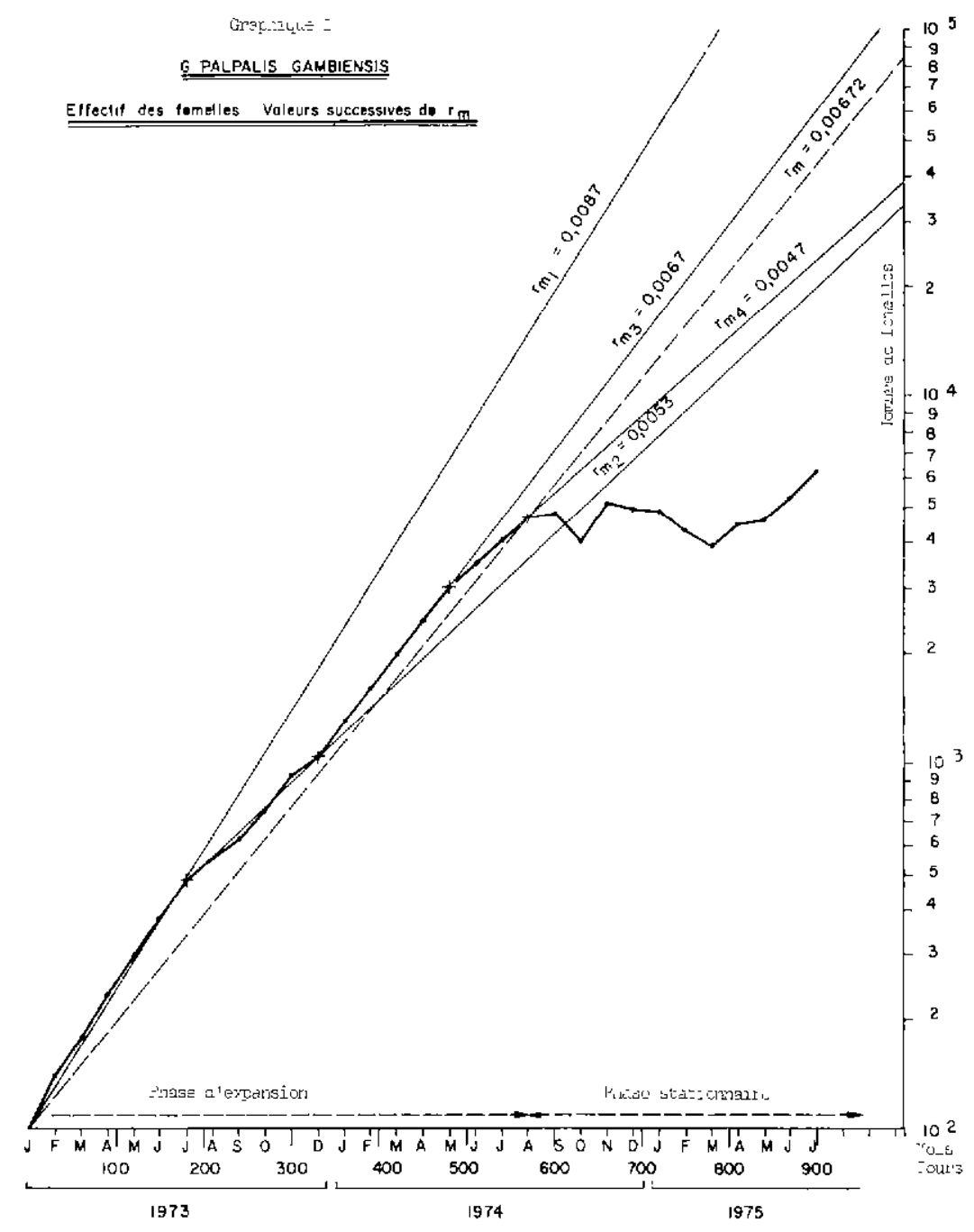

A partir du 15 août 1974, l'élevage est entré dans une phase stationnaire, par prélèvement de pupes et d'adultes en excédent, à raison de 3000 individus par mois. L'effectif des femelles en élevage a ainsi été maintenu à une moyenne de 4700 femelles environ jusqu'à la fin de l'année 1974.

\section{CARACTÉRISTIQUES BIOLOGIQUES DE LA SOUCHE EN ÉLEVAGE}

\subsection{Mortalité des femelles avant ou pendant l'accouplement}

La mortalité des femelles non reproductrices (ensemble des femelles mortes avant d'être accouplées et des femelles trouvées mortes à la séparation des sexes après l'accouplement), pendant la phase d'expansion de l'élevage (du 26 juillet 1972 au 14 août 1974) a été de 14,19 p. 100.
Elle a varié, suivant les périodes définies précédemment, entre 10,79 et 21,01 p. 100 , le pourcentage le plus élevé se situant pendant la phase initiale de démarrage ( $\mathrm{I}^{\text {re }}$ période) :

$1^{\text {re }}$ période : 58 femelles mortes pour 276 écloses $(21,01$ p. 100$)$,

$2^{\mathrm{e}}$ période : 119 femelles mortes pour 1038 écloses $(11,46$ p. 100$)$,

$3^{\mathrm{e}}$ période : 226 femelles mortes pour 2093 écloses $(10,79$ p. 100$)$,

$4^{\mathrm{e}}$ période : 736 femelles mortes pour 5529 écloses $(13,31$ p. 100),

$5^{\text {e }}$ période : 1114 femelles mortes pour 6932 écloses $(16,07$ p. 100).

\subsection{Taux d'insémination}

L'examen des spermathèques de 125 femelles accouplées à l'âge de 3 à 4 jours, avec des mâles 
âgés de 7 à 10 jours, puis séparées des mâles $48 \mathrm{~h}$ après l'accouplement, et disséquées 2 jours plus tard, donne un pourcentage d'insémination supérieur à 98 p. 100 :

- spermathèques pleines : 116 femelles (92,8 p. 100$)$,

- spermathèques presque pleines : 5 femelles (4,0 p. 100),

- spermathèques à demi pleines : 2 femelles (1,6 p. 100), 100).

- spermathèques vides: 2 femelles $(1,6 \mathrm{p}$.

\subsection{Longévité des femelles après accouplement et production de pupes}

Les longévités et productions de pupes des femelles reproductrices ont été calculées à partir d'un échantillonnage de plusieurs lots de femelles pris dans chacune des 5 périodes de la phase d'expansion.

Afin de pouvoir comparer les différents lots entre eux, les nombres réels de femelles au jour 0 (moyenne des dates d'éclosion des femelles vivantes après l'accouplement dans chaque lot) ont été ramenés à 100 femelles.
Compte tenu du nombre moyen de femelles vivantes, à chaque décade, et du nombre de pupes produites, on obtient ainsi les pourcentages mensuels de femelles vivantes et le nombre de pupes produites en 30 jours pour 100 femelles initiales figurant dans le tableau II.

En moyenne (avant dernière colonne du tableau), plus de la moitié des femelles vivent au-delà de 100 jours, la longévité maximale dépasse 200 jours et chaque femelle produit au cours de sa vie 4,7 pupes.

Ces chiffres sont nettement améliorés dans les conditions de l'élevage individuel.

C'est ainsi que 64 femelles écloses les 5 et 6 mars 1974 (au cours de la $5^{\mathrm{e}}$ période), et mises, après accouplement, en petites cages individuelles, ont eu une longévité moyenne de 107 jours. La longévité la plus faible a été de 13 jours et la plus longue de 300 jours.

L'ensemble de ces femelles a produit 483 pupes, soit 7,5 pupes par femelle, mais 4 femelles n'ayant produit aucune pupe, la production moyenne des femelles pondeuses a été en fait de 8 pupes par femelle. Les productions maximales ont été de 22 pupes ( 1 femelle morte au $258^{\circ}$ jour) et de 25 pupes ( 1 femelle morte au $261^{\mathrm{e}}$ jour).

TABLEaU N*II - G. p. Bambiensis - Longévités des femelles et productions de pupes

\begin{tabular}{|c|c|c|c|c|c|c|c|c|}
\hline Périodes & & $\begin{array}{cc} & \text { lère } \\
\text { du } & 26.7 .1972 \\
\text { au } & 21.1 .1973\end{array}$ & $\begin{array}{cc} & 2 \mathrm{e} \\
\mathrm{du} & 22.1 .73 \\
\mathrm{au} & 20.7 .73\end{array}$ & $\begin{array}{c}3 e \\
\text { du } 21.7 .73 \\
\text { au } 17.12 .73\end{array}$ & $\begin{array}{c}4 \mathrm{e} \\
\text { du } 18.12,73 \\
\text { au } 16.5 .73\end{array} \mid$ & $\begin{array}{cc} & 5 \mathrm{e} \\
\text { du } & 17.5 .73 \\
\text { au } & 14.8 .74\end{array}$ & Moyenne & $\begin{array}{c}\text { Elevage } \\
\text { individuel }\end{array}$ \\
\hline $\begin{array}{l}\text { Nombres rëels } \\
\text { de femelles } \\
\text { accouplées dans } \\
\text { chacun des } \\
\text { échantillons }\end{array}$ & & 88 & 158 & 663 & 561 & 622 & 2092 & 64 \\
\hline \multirow{3}{*}{$\begin{array}{l}\text { P. } 100 \mathrm{de} \\
\text { feme } 11 \mathrm{es}\end{array}$} & $30 \mathrm{j}$ & 85,2 & 82,9 & 79,3 & 90,0 & 79,9 & 82,9 & 79,6 \\
\hline & $60 \mathrm{j}$ & 62,5 & 66,4 & 60,2 & 75,9 & 63,1 & 65,9 & 65,6 \\
\hline & $90 \mathrm{j}$ & 40,9 & 41,7 & 38,7 & 58,2 & 40,9 & 44,9 & 53,1 \\
\hline \multirow{3}{*}{ vivantes } & $120 j$ & 18,2 & 22,1 & 23,9 & 34.9 & 18,8 & 25,0 & 42,1 \\
\hline & $150 \mathrm{j}$ & 12,5 & 10,1 & 9,3 & 14,4 & 7,0 & 10,2 & 23,4 \\
\hline & $180 \mathrm{j}$ & 4,5 & 1,9 & 2,7 & 3,4 & 1,4 & 2,5 & 12,5 \\
\hline \multirow{6}{*}{$\begin{array}{l}\text { Nombres de pupes } \\
\text { produites p. } 100 \\
\text { o initiales }\end{array}$} & $30 \mathrm{j}$ & 101 & 97 & 56 & 69 & $B 1$ & 72 & 130 \\
\hline & $60 j$ & 292 & 271 & 198 & 239 & 232 & 228 & 309 \\
\hline & $90 \mathrm{~J}$ & 438 & 402 & 313 & 386 & 346 & 355 & 461 \\
\hline & $120 j$ & 512 & 487 & 386 & 495 & 401 & 433 & 587 \\
\hline & $150 \mathrm{j}$ & 537 & 515 & 417 & 544 & 423 & 465 & 675 \\
\hline & $180 \mathrm{j}$ & 545 & 521 & 425 & 557 & 425 & 473 & 720 \\
\hline
\end{tabular}


4 femelles n'ont produit aucune pupe

19 femelles ont produit de 1 à 3 pupes

9 femelles ont produit de 4 à 6 pupes

7 femelles ont produit de 8 à 9 pupes

13 femelles ont produit de 10 à 12 pupes

8 femelles ont produit de 13 à 16 pupes

4 femelles ont produit de 18 à 25 pupes

\subsection{Rythme de ponte}

Dans les conditions de l'élevage, la première larve est pondue entre le $15^{\circ}$ et le $21^{\mathrm{e}}$ jour qui suit l'éclosion de la femelle (moyenne sur 44 femelles parmi les 60 femelles pondeuses suivies individuellement $=17,6$ jours).

Les observations portant sur 345 périodes séparant 2 pontes successives sans avortement donnent une durée interlarvaire moyenne de 9,8 jours (minimum $=6$ jours ; maximum $=$ 13 jours).

Des irrégularités dans les délais successifs de ponte peuvent se produire, généralement par émission, à un stade très précoce, d'une larve non viable. Les intervalles séparant alors la ponte de 2 larves normales sont en moyenne de 14,4 jours, soit un raccourcissement de près de 3 jours entre 2 ovulations successives.

\subsection{Poids des pupes}

Trois séries de pesées, réalisées dans des conditions identiques, (pupes âgées de moins de $24 \mathrm{~h}$; balance de précision sensible au 1/100 de $\mathrm{mg}$; conditions d'environnement semblables) ont été effectuées à 3 époques différentes :

a) Les pesées effectuées sur 592 pupes pondues entre le 22 mai et le 20 juin 1973 , par des femelles élevées en groupe, donnent un poids moyen de $22,69 \pm 0,32 \mathrm{mg}$.

439 pupes $(74,1$ p. 100$)$ pèsent plus de $20 \mathrm{mg}$ (poids moyen $=24,42 \pm 0,27 \mathrm{mg}$ ) et 153 pupes $(25,8$ p. 100$)$ pèsent moins de $20 \mathrm{mg}$ (poids moyen $=17,71 \pm 0,31 \mathrm{mg}$ ).

b) Le poids moyen de 475 pupes produites par les femelles du lot 05.03.74 élevées individuellement est de 21,61 $\pm 0,36 \mathrm{mg}$.

326 pupes $(68,6 \mathrm{p} .100)$ pèsent plus de $20 \mathrm{mg}$ (poids moyen $=23,75 \pm 0,27 \mathrm{mg}$ ), et 149 pupes (31,3 p. 100) pèsent moins de $20 \mathrm{mg}$ (poids moyen $=16,92 \pm 0,34 \mathrm{mg}$ ). c) Le poids moyen de 310 pupes, pondues le 16 décembre 1975, par des femelles élevées en groupe, est de $23,91 \pm 0,40 \mathrm{mg}$.

273 pupes $(88,06$ p. 100$)$ pèsent plus de $20 \mathrm{mg}$ (poids moyen $=24,76 \pm 0,35 \mathrm{mg}$ ) et $37(11,93$ p. 100) moins de $20 \mathrm{mg}$ (poids moyen $=17,64 \pm$ $0,55 \mathrm{mg}$ ).

Alors que les proportions entre les pupes légères et les pupes lourdes diffèrent de façon peu significative entre les lots $a$ ) et $b$ )

$$
\left(X^{2}=3,961-\text { d. d. } 1 .=1\right),
$$

cette différence est très significative entre les lots a) et $c)\left(\mathrm{X}^{2}=23,671-\right.$ d. d. $\left.1 .=1\right)$.

Les moyennes des poids diffèrent également de façon très significative entre les 3 lots (lots $a$ ) et b) $: \varepsilon=4,691-\operatorname{lots} a$ ) et $c$ ) $: \varepsilon=4,919$ ).

Le poids des pupes du lot $a$ ), significativement moins élevé que celui du lot $c$ ), est par contre plus élevé que celui des pupes de $1^{\text {re }}$ génération (différence significative $: \varepsilon=2,64$ ).

Il y a donc eu, au cours du temps, augmentation du poids des pupes en même temps qu'une proportion plus importante pesait plus de $20 \mathrm{mg}$.

Assez curieusement, les femelles élevées individuellement pondent des pupes plus légères que les femelles élevées en groupe.

Une comparaison entre le poids moyen de pupes ayant donné naissance à des mâles et le poids moyen de pupes ayant donné naissance à des femelles, a en outre été effectuée, à partir de pupes de plus de $20 \mathrm{mg}$ pondues par des femelles élevées individuellement :

- les pupes mâles $(n=97)$ ont un poids moyen de 24,58 $\pm 0,52 \mathrm{mg}$,

- les pupes femelles $(n=89)$ ont un poids moyen de $25,10 \pm 0,60 \mathrm{mg}$.

Il n'y a pas de différence significative entre ces deux moyennes $(\varepsilon=1,279)$.

CHALLIER (2) trouve au contraire une différence significative entre les pupes mâles (poids moyen $22,77 \mathrm{mg}$ ) et les pupes femelles (poids moyen $24,63 \mathrm{mg}$ ) qu'il a élevées en Haute-Volta.

\subsection{Durées de nymphoses}

Les durées du stade pupal, calculées sur 287 mâles et 345 femelles éclos entre le 19 mars et le 2 avril 1974 , sont de $37,86 \pm 0,15$ jours pour les mâles, avec des écarts de 35 à 40 jours, et de $35,46 \pm 0,15$ jours pour les femelles, avec des écarts de 32 à 39 jours. 
Par comparaison avec les individus de première génération, il y a eu allongement des durées de nymphose de près d'un jour pour les femelles (différence significative à 1 p. 100) et d'un peu plus d'un jour pour les mâles (différence significative à 1 p. 10000 ).

\subsection{Pourcentages d'éclosion et sex-ratio}

On trouvera, dans le tableau I, $4^{e}$ colonne, les taux d'éclosion correspondant à chacune des périodes de 30 jours de la phase d'expansion. Ces pourcentages ont été calculés à partir des nombres bruts d'individus éclos, qu'ils aient, ou non, survécus plus de $24 \mathrm{~h}$ après l'éclosion.

Le pourcentage réel d'éclosion des individus viables (ayant vécu plus de $24 \mathrm{~h}$ ), pour l'ensemble de la période comprise entre le 25 août 1972 et le 14 août 1974, est de 94,07 p. 100 (40 577 pupes conservées dont sont éclos 19340 mâles et 18831 femelles).

Le sex-ratio est en faveur des mâles; il éclôt en effet 50,66 p. 100 de mâles contre 49,33 p. 100 de femelles. Par contre, la longévité des mâles est inférieure à celle des femelles (longévité moyenne des mâles $=50$ jours environ ; longévité moyenne des femelles $=100$ jours environ).

\section{L'ÉLEVAGE EN PHASE STATIONNAIRE}

\section{PRODUCTIVITÉ GLOBALE}

La souche de G.p. gambiensis de MaisonsAlfort est entrée dans la phase stationnaire, par retrait des pupes et femelles adultes en excédent, à partir du 15 août 1974.

Entre cette date et le 8 septembre 1975, soit 390 jours, l'effectif a été maintenu à une moyenne de 4800 femelles/jour.

Le taux moyen de mortalité de ces femelles n'a pas dépassé 1,5 p. 100 par jour.

La production moyenne, en 30 jours, a été de 1,66 pupes par femelle, soit 8000 pupes environ par mois (104 500 pupes en 390 jours).

Le pourcentage moyen d'éclosion des pupes conservées atteint 95,5 p. 100.

La production excédentaire globale a été de 38800 pupes (700 pupes environ par semaine) et de 2400 femelles adultes (environ 180 femelles par mois), qui ont été distribuées à divers laboratoires ou organismes de recherche, français ou étrangers.

\section{PRODUCTIVITÉ OPTIMALE}

Deux lots de femelles, l'un ( $\operatorname{lot} \mathrm{n}^{0} 29.11 .73$ ) comprenant 177 femelles élevées en groupe, l'autre (lot $n^{\circ} 05.03 .74$ ) comprenant 64 femelles élevées individuellement, choisis en fonction des durées de vie, rythme de ponte et quantités de pupes obtenues, ont servi à calculer, suivant la méthode décrite par CURTIS et JORDAN (4), et JORDAN et CURTIS $(9,10)$, la productivité optimale de G.p. gambiensis dans les conditions d'élevage réalisées à Maisons-Alfort.

Dans ces conditions, la durée moyenne de nymphose des femelles est de 35 jours environ. La première larve est pondue lorsque la femelle adulte est âgée de 18 jours et les périodes interlarvaires suivantes sont proches de 10 jours. Les pourcentages d'éclosion et les mortalités des jeunes femelles non reproductrices ont été respectivement de 93,6 p. 100 et 8,29 p. 100 pour le lot 29.11 .73 et de 95,0 p. 100 et 12,32 p. 100 pour le lot 05.03 .74 .

\section{1. Etablissement des tables de vie et calcul du coefficient d'accroissement}

Dans les calculs ayant servi à l'établissement des tables de vie (tabl. III et IV), la longévité des femelles, depuis la ponte à l'état larvaire jusqu'à la mort des dernières femelles reproductrices, a été divisée en périodes $\left(\mathrm{g}_{\mathrm{x}}\right)$ de 10 jours numérotées de 0 à $\mathbf{n}$.

Les 3 premières $(0,1,2)$ correspondent à la vie pupale. Les éclosions se produisent dans le groupe d'âge $g_{x} 3$; la classe $g_{x} 4$ ne comprend que des jeunes femelles non reproductrices et les pontes (femelles reproductrices) commencent à partir de $g_{x} 5$.

On considère qu'il n'y a pas de mortalité pendant la vie pupale $\left(g_{x} 0\right.$ à $\left.g_{x} 2\right)$. Les mortalités correspondantes et la mortalité à l'éclosion sont rassemblées dans le groupe d'âge $g_{\mathrm{x}} 3$. Dans la classe $g_{x} 4$, on tient compte de la mortalité des jeunes femelles avant la ponte. A partir de $g_{x} 5$, figurent les proportions de femelles reproductrices survivantes dans chacun des groupes d'âge correspondants.

On considère en outre que le sex-ratio est de $1 / 1$ à la ponte ; la fécondité spécifique $\left(\mathrm{m}_{\mathrm{x}}\right)$, qui est exprimée en larves femelles, est donc obtenue en divisant la moitié du nombre total des pupes produites pendant la période $\mathrm{g}_{\mathrm{x}}$ correspondante 
1ABL. $N^{0} I I I-G$, patpalis gambiensis. Table de vie. Lot 05.03 .74 (64 q élevêes individuellement)

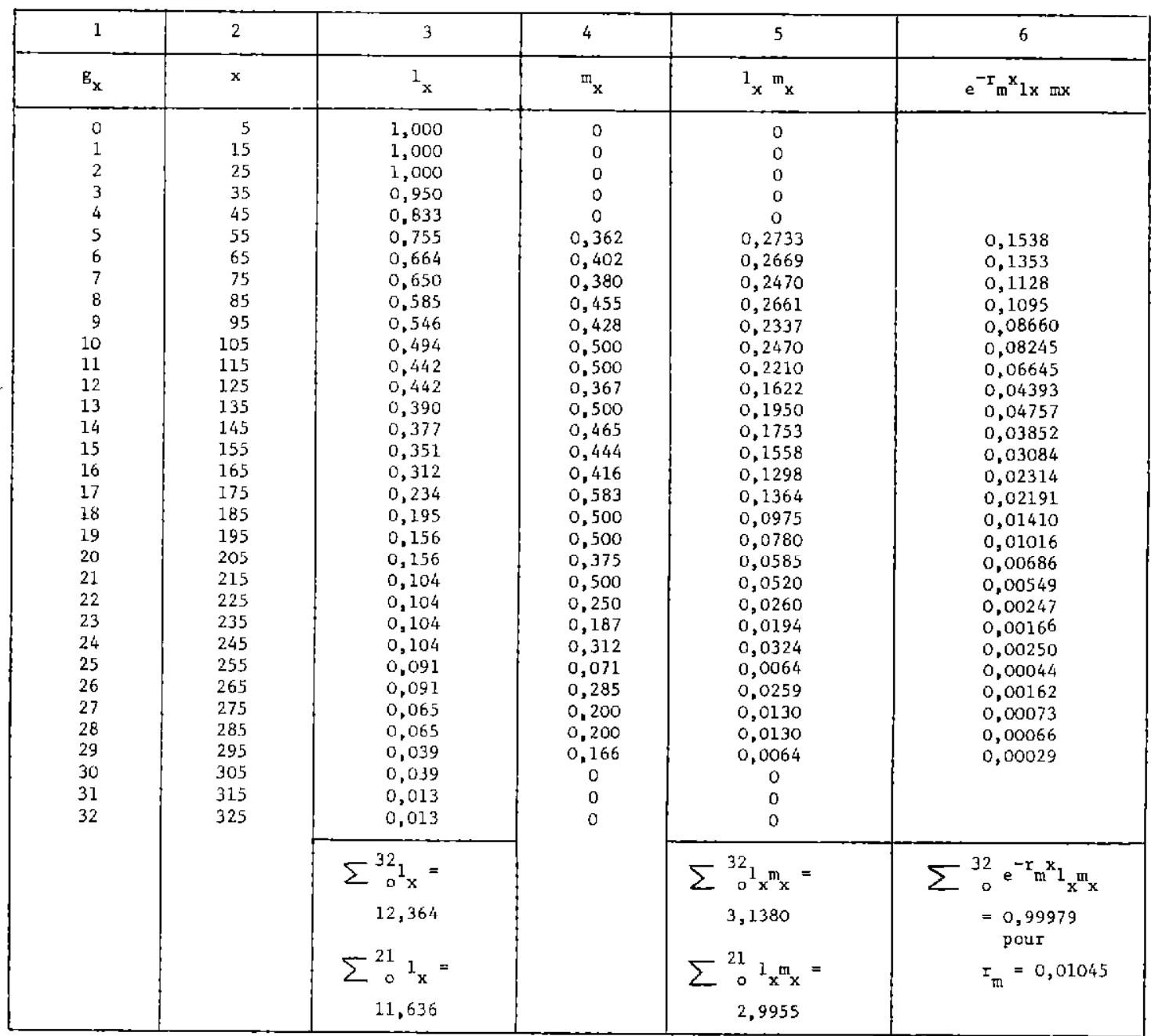

$\varepsilon_{x}=$ classe d'âge ; $x=$ âge central de la classe correspondante ;

$l_{x}=$ probabilité de survie des fenelles $; \mathbb{m}_{x}=$ fécondité spêcifique.

(l'unité de temps de $\mathrm{r}_{\mathrm{m}}$ est 1 jour et les valeurs de $\mathbf{x}$ sont exprimées en jours).

par le nombre de femelles survivant pendant cette période.

Les longévités des femelles des deux lots ayant servi à cette étude et leurs fécondités spécifiques sont représentées sur les graphiques 3 et 4 . On constate que la fécondité des femelles reproductrices est pratiquement constante et proche de 0,45 pour le lot 05.03 .74 et de 0,40 pour le lot 29.11.73, jusqu'au groupe d'âge 21 ( 215 jours). Elle chute ensuite, dans le premier lot, à une valeur inférieure à 0,25 . Ceci signifie que, jusqu'au groupe d'âge 21,80 à 90 p. 100 des femelles produisent une larve à chaque cycle d'ovulation.

Le coefficient d'accroissement $r_{m}$ a été calculé à partir des tables de vie établies pour chacun des deux lots (tabl. III et IV), par essais-erreurs des différentes valeurs possibles de $r_{m}$ telles que

$$
\sum_{0}^{n} e^{-r_{m} x} I_{x} m_{x}=1 .
$$

Par cette méthode, on obtient les valeurs suivantes de $r_{m}$ :

- femelles élevées individuellement (lot $05.03 .74)$

$$
r_{m}=0,01045
$$

— femelles élevées en groupe (lot 29.11.73)

$$
r_{m}=0,0095 \text {. }
$$




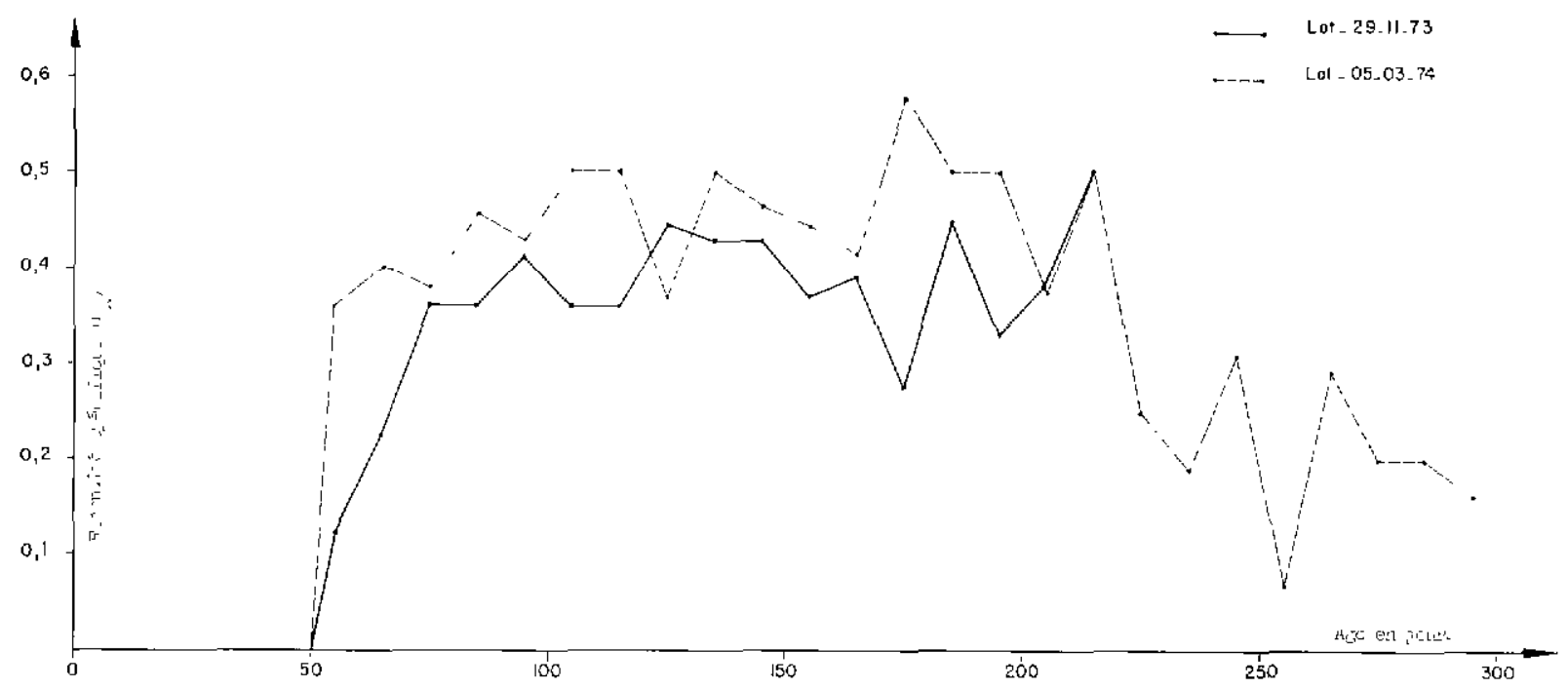

Dans le tableau $\mathrm{V}$, on a indiqué le temps de doublement, qui est un facteur constant dans le cas d'une croissance exponentielle, des effectifs dans des élevages en phase d'expansion (colonne 2) où $r_{m}$ aurait les valeurs calculées ci-dessus.

On a également fait figurer dans ce tableau (colonne 3) les facteurs d'accroissement des effectifs en un an.
Les courbes de croissance exponentielle correspondantes sont représentées sur le graphique 5 , où l'axe des ordonnées (effectif des femelles) est à l'échelle logarithmique. Par comparaison, on a également tracé sur ce graphique les courbes de croissance correspondant aux valeurs de $r_{m}$ obtenues à partir de la courbe réelle des effectifs pendant la $2^{\mathrm{e}}$ période $\left(\mathrm{r}_{\mathrm{m}}=0,0087\right)$ et pendant la totalité de la phase d'expansion $\left(r_{\mathrm{m}}=0,00672\right)$

TABLEAU $\mathrm{N}^{\circ} \mathrm{V}-G \cdot p \cdot$ gambiensis.

\begin{tabular}{|c|c|c|c|c|c|}
\hline & & & $\begin{array}{c}\text { Elevage individue } 1 \\
(10 \mathrm{t} 05.03 .74)\end{array}$ & $\begin{array}{c}\text { Elevage } \\
\text { en groupe } \\
\text { (1ot } 29.11 .73 \text { ) }\end{array}$ & $\begin{array}{c}\text { Courbe réelle } \\
\text { des effectifs } \\
\text { en phase } \\
\text { d'expansion }\end{array}$ \\
\hline $\begin{array}{l}\text { Colonie } \\
\text { en phase } \\
\text { d'expansion }\end{array}$ & & $\begin{array}{l}\text { (1) Valeur de } r \\
\text { (2) Temps de doublement } \\
\text { en jours } \\
\text { (3) Accroissement en } 1 \text { an }\end{array}$ & $\begin{array}{c}0,01045 \\
66,3 \\
45 \text { fois }\end{array}$ & $\begin{array}{l}0,0095 \\
72,9 \\
32 \text { fois }\end{array}$ & $\begin{array}{l}0,00672 \\
103,4 \\
12 \text { fois }\end{array}$ \\
\hline \multirow{4}{*}{$\begin{array}{l}\text { Colonie } \\
\text { en phase } \\
\text { stationnaire }\end{array}$} & \multirow{2}{*}{ 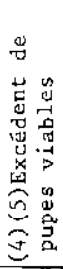 } & $\begin{array}{l}\text { Quantité hebdomadaire de } \\
\text { pupes de chaque sexe pour } \\
\text { une colonie de } \mathrm{N} \\
\text { mouches adultes }\end{array}$ & $0,157 \mathrm{~N}$ & $0,119 \mathrm{~N}$ & \\
\hline & & $\begin{array}{l}\text { Dimension de la colonie } \\
\text { pour une production excé- } \\
\text { dentaire de } 10 \text { ooo pupes } \\
\text { de chaque sexe par semaine }\end{array}$ & 64 000 adultes & 84000 adultes & \\
\hline & \multirow{2}{*}{ 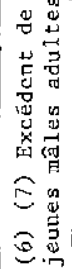 } & $\begin{array}{l}\text { Quantıté hebdomadaire de } \\
\text { mâles disponibles pour } \\
\text { une colonie de N } \\
\text { mouches adultes }\end{array}$ & $0,205 \mathrm{~N}$ & $0,162 \mathrm{~N}$ & \\
\hline & & $\begin{array}{l}\text { Dimension de la colonie } \\
\text { pour une production excé- } \\
\text { denta1re de } 5 \text { 000 (ou } \\
\text { lo } 000 \text { ) mâles par semaine }\end{array}$ & $\begin{array}{l}25000 \text { adultes } \\
\langle 49000 \text { adultes }\rangle\end{array}$ & $\begin{array}{l}31000 \text { adultes } \\
\text { (62 000 adultes) }\end{array}$ & \\
\hline
\end{tabular}




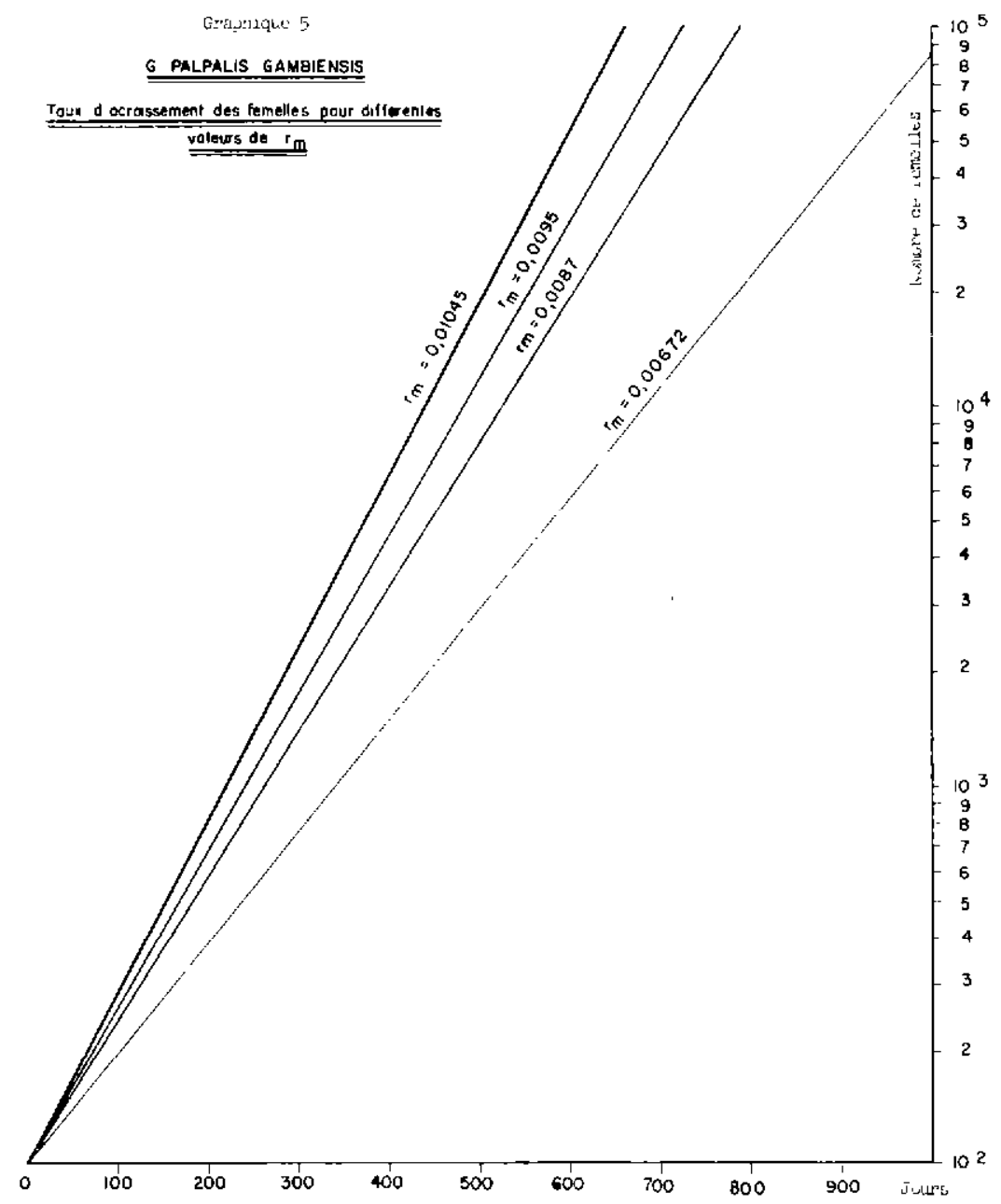

2.2. Productivité optimale de l'élevage en phase stationnaire

Les nombres d'individus, à l'état pupal ou à l'état adulte, disponibles dans des élevages de G.p.gambiensis en phase stationnaire, pour lesquels les coefficients d'accroissement $r_{m}$ ont les valeurs calculées précédemment $\left(r_{m}=0,01045\right.$ et $\left.r_{m}=0,0095\right)$, ont été obtenus par la méthode de MONRO et OSBORN (12) adaptée par CURTIS et JORDAN (4) à leurs élevages de glossines.

Dans le cas des élevages de G.p.gambiensis, on a tenu compte de ce que les périodes interlarvaires sont de 10 jours et on a estimé que les femelles reproductrices étaient tuées après le groupe d'âge 21. On a également supposé que les mâles conservés comme reproducteurs sont accouplés une première fois à l'âge de 7 jours (pendant le groupe d'âge $g_{x} 4$ ), à nouveau 2 fois pendant le groupe d'âge $\mathrm{g}_{x} 5$ et qu'ils sont tués à la fin du groupe d'âge 5. Chaque mâle "étalon » est ainsi conservé pendant 2 unités de temps pendant lesquelles il est accouplé 3 fois. La moitié de ces mâles doit être remplacée à chaque unité de temps. Le nombre de mâles étalons à conserver, sans tenir compte des mortalités, sera donc:

$\frac{2}{3} \times \frac{1}{2}=\frac{1}{3}$ du nombre de mâles éclos

au groupe d'âge 3 .

A partir d'un élevage en phase stationnaire de $\mathrm{N}$ mouches adultes, les nombres de pupes de chaque sexe disponibles par semaine seront obtenus en résolvant l'équation :

$$
\frac{\frac{7}{10} \mathrm{NJ}_{3}\left[\sum_{0}^{21}\left(\mathrm{l}_{\mathrm{x}} \mathrm{m}_{\mathrm{x}}\right)-1\right]}{\sum_{3}^{21} \mathrm{l}_{\mathrm{x}}} .
$$


et le nombre de jeunes mâles adultes disponibles chaque semaine sera donné par l'équation:

$$
\frac{\frac{7}{10} N 1_{3}\left[\sum_{0}^{21}\left(1_{x} m_{x}\right)-\frac{1}{3}\right]}{\sum_{3}^{21} 1_{x}}
$$

dans lesquelles

$$
\mathrm{l}_{3}, \sum_{0}^{21}\left(\mathrm{l}_{\mathrm{x}} \mathrm{m}_{\mathrm{x}}\right) \quad \text { et } \quad \sum_{3}^{21} \mathrm{l}_{\mathrm{x}}
$$

sont obtenus à partir des tables de vie des tableaux III et IV.

Les valeurs correspondantes ainsi calculées figurent dans le tableau $V$.

On a en outre indiqué dans ce tableau les nombres d'individus adultes qu'il sera nécessaire d'élever pour avoir une production excédentaire soit de 10000 pupes de chaque sexe par semaine ( $5^{\mathrm{e}}$ colonne), soit de 5000 (ou 10000 ) jeunes mâles adultes par semaine ( $7^{\mathrm{e}}$ colonne).

\section{CONCLUSIONS}

CHALLIER (1) et LAVEISSIÈRE (11) ont montré les difficultés d'élevage, en Afrique, de G.p. gambiensis. CHALLIER estime que les conditions d'élevage en laboratoire favorisent la survie des individus de petite taille et des individus chétifs dont la multiplication provoque, au bout de quelques générations, l'extinction de l'élevage.

LAVEISSIÈRE préconise, pour augmenter la production et la taille des pupes, d’élever cette espèce dans l'obscurité totale.

Les techniques utilisées à Maisons-Alfort ont toutefois permis à la souche de G.p. gambiensis en élevage de dépasser la $20^{\mathrm{e}}$ génération tout en étant maintenue à un effectif élevé.

Le poids des pupes produites au laboratoire constitue un bon critère de la qualité d'un élevage. Bien que nous ne disposions pas de données sur le poids des pupes sauvages de G. p. gambiensis, il semble raisonnable d'estimer que, par comparaison avec le poids des pupes pondues au laboratoire par les femelles sauvages, le poids des pupes de l'élevage de Maisons-Alfort se rapproche de celui des pupes pondues dans la nature.

Le fait que le poids des pupes pondues au laboratoire ait augmenté de façon significative au cours du temps, pendant que la proportion de pupes légères allait en diminuant, peut en tout cas être interprété comme un signe de la bonne qualité de cet élevage.

CURTIS et JORDAN (4), ont calculé les coefficients d'accroissement, dans les conditions d'élevage réalisées à Langford, de G. austen $\left(\mathrm{r}_{\mathrm{m}}=0,0173\right)$ et de $G$. morsitans $\left(\mathrm{r}_{\mathrm{m}}=0,0166\right)$ nourries sur lapin. A Lisbonne, PINHAO (13) a trouvé une valeur de $r_{m}$ de 0,0123 avec son élevage de $G$. morsitans nourrie sur cobaye.

Avec les espèces du groupe palpalis élevées individuellement à Anvers, VAN DER VLOEDT (14) obtient les coefficients suivants :

$$
\begin{aligned}
& \text { G.p.palpalis : }: \mathrm{r}_{\mathrm{m}}=0,0092 \\
& \text { G.f.quanzensis : } \mathrm{r}_{\mathrm{m}}=0,0077 .
\end{aligned}
$$

Les résultats obtenus avec la souche de G. p. gambiensis élevée à Maisons-Alfort sont en accord avec ceux obtenus à Anvers et semblent confirmer que l'élevage des espèces du groupe palpalis, à l'exception de G. tachinoides (8), est plus délicat que celui des espèces du groupe morsitans.

Ces résultats, qui ont été obtenus à partir d'individus élevés en grand nombre, avec des méthodes simples et standardisées, peuvent certainement être améliorés dans des conditions d'élevage en petites unités expérimentales dans lesquelles il est possible d'apporter plus de soin à chaque individu.

Les buts qui sont poursuivis à Maisons-Alfort visent au contraire à obtenir, à partir de méthodes applicables aux conditions difficiles du continent africain, des élevages importants permettant de disposer d'un nombre élevé de mâles utilisables dans des campagnes de lutte génétique.

A partir des données figurant dans le tableau $\mathrm{V}$ il est possible de faire, dans ces conditions, des prévisions pour l'établissement en Afrique d'un élevage de G. p. gambiensis.

Le programme établi pour le Centre I. E. M. V. T. de Bobo-Dioulasso prévoyait dans un premier temps la création d'un élevage de 30000 femelles permettant une production de 20000 mâles stériles par mois, soit 5000 mâles par semaine (3). Les résultats obtenus à MaisonsAlfort montrent que ces prévisions étaient réalistes, et que, dans l'optique la moins favorable (coefficient $r_{m}=0,00672$ ), l'effectif des 30000 femelles peut être obtenu, avec 100 femelles seulement au départ, en 850 jours, soit un peu plus de 2 ans, et, dans le cas le plus favorable $\left(r_{\mathrm{r}}=0,01045\right)$, en 550 jours, soit un an et demi. 


\section{SUMMARY}

The rearing of Glossine palpalis gambiensis Vanderplank, 1949 (Diptera-Muscidae) at Maisons-Alfort

A colony of Glossina palpals gambiensis was begun in July 1972, at the I. E. M. V. T. entomology laboratory in Ma1sons-Alfort (France), starting from 126 parental females, which emerged from pupae and wild females collected in Upper-Volta.

This colony was established in order to study, under laboratory conditions, the biology of that species and the conditions of mass rearing in view of an application, in Upper-Volta, of the control by the sterile male technique.

The author sums up the results gained from the beginning of the rearing to September 1975. The strain has followed an expanding phase for two years (August 1972-August 1974) with a mean rate of increase in numbers of females $\left(\mathrm{r}_{\mathrm{m}}\right)$ of 0,00672 . Since August 1974, the number of flies is maintained at a constant size of about 4800 females by withdrawing the surplus of offspring (about 3000 pupae and 180 females per month).

On the basis of these results, predictions can be made for the establishment of a colony of that species in Africa.

\section{RESUMEN}

\section{La cria de Glossina palpalis gambiensis Vanderplank, 1949 \\ (Diptera-Muscidae) en Maisons-Alfort}

En julio de 1972, se ha empezado una cria de Glossina palpalis gambiensis en el laboratorio de entomologia del I. E. M. V. T., en Maisons-Alfort (Francia) a partir de 126 hembras nacidas de pupas y hembras salvajes recogidas en Altd-Volta.

Se creó dicha cria con el fín de estudiar, en laboratorio, la biologia de esta especie y las condiciones de producción de una cria importante para aplicar, en Alta-Volta, la técnica de lucha por el soltar de machos esteriles.

El autor indica los resultados obtenidos desde el principio de la cria hasta septiembre de 1975. Durante dos años (de agosto de 1972 a agosto de 1974) la cepa ha sido en « fase de expansión » con un coeficiente de aumento medio de 0,006 72. Desde agosto de 1974, el número medio de moscas sigue siendo constante, sea unas 4800 hembras, por toma de las pupas o adultos excedentes (unas 3000 pupas y 180 hembras por mes).

Estos resultados permiten hacer previsiones para el establecimiento de una cria importante de dicha especie en Africa.

\section{BIBLIOGRAPHIE}

1. CHALLIER (A.). L'élevage de Glossina palpalis gambiensis Vanderplank, 1949 en Haute-Volta. Criação de mosca tsé-tsé em laboratorio e sua aplicação pratica. $1^{\circ}$ Symp. intern. 1969. Coordenação de J. Fraga de Azevedo. Lisboa 1970, pp. 85-90.

2. CHALLIER (A.). Ecologie de Glossina palpals gambiensis Vanderplank, 1949 (Diptera-Muscidae) en savane d'Afrique occidentale. Mém. O. R. S. T. O. M., Paris, 1973, 64, 274 p.

3. CLAIR (M.). Projet de lutte contre les mouches tsétsé par lâchers de mâles stértles. Rev. Elev. Méd. vét. Pays trop., suppl. (Les moyens de lutte contre les trypanosomes et leurs vecteurs. Actes du colloque Paris, mars 1974), pp. 127-129.

4. CURTIS (C. F.) et JORDAN (A. M.). Calculations of the productivity of Glossina austeni Newst. maintained on goats and on lop-eared rabbits. Bull. ent. Res., 1968, 59 : 651-658.

5. ITARD (J.). Elevage, cytogénétique et spermatogénèse des inseztes du genre Glossina. Stérilisation des mâles par irradiation gamma. Ann. Parasit. hum. comp., 1971, 46 (3 bis) : 35-66.

6. ITARD (J.). Techniques d'élevage des glossines. Perspectives offertes pour l'utilisation de la méthode de lutte par lâchers de mâles stériles. Bull. Off. int Epiz., 1971, 76 : 307-318.

7. ITARD (J.). Situation actuelle des élevages de glossines à 1'I. E. M. V. T. (Maisons-Alfort). Rev Elev. Méd. vét. Pays trop. suppl. (Les moyens de lutte contre les trypanosomes et leurs vecteurs. Actes du colloque Paris, mars 1974), pp. 51-53.

8 ITARD (J.), MAILLOT (L.), BRUNET (J.) et GIRET (M.). Observations sur un élevage de Glossina rachmoides West., après adoption du lapin comme animal hôte. Rev. Elev. Méd. vét. Pays frop., 1968, 21 (3) : 387-403.

9. JORDAN (A. M.) et CURTIS (C. F.). Productivity of $G$. austeni Newst maintained on lop eared rabbits. Bull. ent. Res., 1968, 58 (2) : 399.410.

10. JORDAN (A. M.) et CURTIS (C. F.). Productivity of Glossina morsitans morsitons Westwood maintained in the laboratory, with particular reference to the sterile-insect release method. Bull. Org. mond. Santé, 1972, 46 (1) : 33-38.

11. LAVEISSIËRE (G.). Essais d'amélioratıon de la technique d'élevage de Glossina palpalss gambiensis Vanderplank en Afrique occidentale. Cah. O. R. S. T. O. M., série Ent. méd. Parasit., 1973, 11 (3) : 205-209. 
12. MONRO (J.) et OSBORN (A.). The use of sterile males to control populations of Queensland fruit-fly, Dacus tryoni (Frogg) (Diptera: Tephritidae). I. Methods of mass-rearing, transporting, irradiating and releasing sterile flies. Aust. J. Zool., 1967, 15 : 461-473.

13. PINHAO (R. da C.). The innate capacity of increase of $G$. morsitans under laboratory conditions. Criação de mosca tsé-tsé em laboratorio e sua aplicação pratica. $1^{0}$ Symp. intern. 1969. Coordenação de J. Fraga de Azevedo. Lisboa. 1970, pp. 305-310.

14. VAN DER VLOEDT (A.). L'élevage au laboratorre de Glossina palpalis palpalis et de Glossina fuscipes quanzensis. Rev. Elev. Méd. vét. Pays trop. suppl. (Les moyens de lutte contre les trypanosomes et leurs vecteurs. Actes du colloque Paris, mars 1974), pp. 61-62. 